\title{
Practical Strategies for the Diagnosis and Assessment of Atopic Dermatitis
}

\author{
Lawrence F. Eichenfield, MD,* and Linda F. Stein Gold, MD ${ }^{\dagger}$
}

\section{Abstract}

Atopic dermatitis (AD) has a significant, lifelong clinical impact on affected individuals and has profound effects on quality of life both for patients and their families. The diagnosis usually can be reliably established on the basis of the history and physical examination. In patients with skin of color, blanching of the skin may be helpful to detect erythema, lichenification, follicular accentuation, and hypopigmentation (all of which are more common than in lighter-skinned patients). Once the diagnosis of $A D$ is established, an assessment of severity, persistence, and impact on the patient's and family's life is important as a guide to treatment decisions.

Semin Cutan Med Surg 36(supp2):S36-S38

(c) 2017 published by Frontline Medical Communications

\section{Keywords}

Atopic dermatitis; eczema

$\mathrm{T}$ he prevalence of atopic dermatitis (AD) in the United States is about $3 \%$ to $5 \%$ in the overall population, or approximately 15 million children and adults. The current lifetime prevalence of $\mathrm{AD}$ in childhood is estimated to be $10 \%$ to $15 \%$, which is an increase of $6 \%$ to $10 \%$ over the last 30 years. ${ }^{1-5}$

In addition, $\mathrm{AD}$ prevalence among children differs according to geography. In the United States, prevalence varies markedly by region, from a low of $8.7 \%$ in Florida to a high of $18.05 \%$ in Maryland. ${ }^{6}$ These differences may be the result of environmental and other factors such as sun exposure, baseline humidity, and urban/rural gradients. ${ }^{7}$

\footnotetext{
* Chief, Pediatric and Adolescent Dermatology, Professor of Dermatology and Pediatrics, Rady Children's Hospital, University of California, San Diego School of Medicine, San Diego, California

${ }^{\dagger}$ Director of Dermatology Research, Henry Ford Health System, Detroit, Michigan

Publication of this CME/CE article was jointly provided by University of Louisville, Postgraduate Institute for Medicine, and Global Academy for Medical Education, LLC, and is supported by an educational grant from Anacor Pharmaceuticals, Inc. The authors have received an honorarium for their participation in this activity. They acknowledge the editorial assistance of Joanne Still, medical writer, and Global Academy for Medical Education in the development of this continuing medical education journal article.
}

Lawrence F. Eichenfield, MD, Advisory Board/Speaker: Valeant Pharmaceuticals North America LLC. Consultant: Anacor/Pfizer Inc., Eli Lilly and Company, Genentech, Inc., Otsuka America Pharmaceutical, Inc./Medimetriks Pharmaceuticals, Inc., Sanofi Genzyme/Regeneron Pharmaceuticals, TopMD, Valeant. Investigator: Sanofi Genzyme/Regeneron.

Linda F. Stein Gold, MD, Consultant: Anacor. Grant/Research: Anacor, GlaxoSmithKline. Data Monitoring Committee: Otsuka.

Address reprint requests to: Lawrence F. Eichenfield, MD, Rady Children's Hospital, 8010 Frost Street, Suite 602, San Diego, CA 92123; leichenfield@ucsd.edu

\section{Diagnosis of AD}

The diagnosis of patients with characteristic signs and symptoms of AD usually does not present a clinical challenge. The typical findings include pruritus, erythema, papules/vesicles, xerosis, excoriations, erosions, and, in many cases, lichenification and dyspigmentation. Questioning during the history taking also provides important diagnostic information: Is the rash chronic or atypical (suggesting another diagnosis or the presence of a comorbid condition)? Does the rash flare and remit (the hallmark history in a patient with AD)? Finally, a positive family history for $\mathrm{AD}$ - in either or both parentsincreases the likelihood that a child has AD.

The characteristic, age-related anatomic distribution of AD provides further evidence to support the diagnosis. ${ }^{8,9}$ In infants, the face (particularly the cheeks and chin), trunk, and extensor extremities are the most common sites of involvement, with sparing of the diaper area. In toddlers and older children, the most commonly affected sites are the flexoral areas of the wrists, ankles, and antecubital and popliteal fossae. In adolescents and adults, the wrists, hands, neck, and ankles are typically affected.

Several clinical features may be associated with AD and may suggest or support the diagnosis (Table 1), although their presence is not specific for $\mathrm{AD} .{ }^{10}$

\section{Differential Diagnosis}

In the few cases in which the diagnosis of AD cannot be determined clinically, a number of other common disorders may be considered in the differential diagnosis (Table 2).

Seborrheic dermatitis (cradle cap) is particularly common in infants from birth to 6 months of age, and represents a potentially confounding finding, as seborrheic dermatitis and eczematous dermatitis often cannot be differentiated in this age group. Diffuse cradle cap in the presence of eczematous dermatitis on the arms and cheeks may resolve over time or may evolve into $\mathrm{AD}$, despite eventual clearance of the cradle cap.

\section{TABLE 1 Findings Possibly Associated With} Atopic Dermatitis

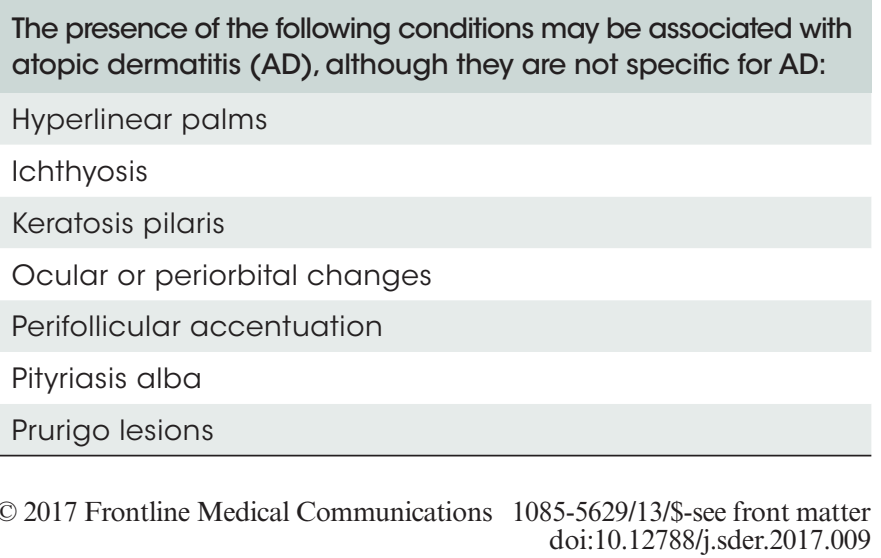


Contact dermatitis is an alternative diagnosis, or it may be a comorbid condition. Careful questioning of the caregiver regarding exposure to potential irritants can help determine whether contact dermatitis might be the culprit or a contributor, and contact allergy testing may be necessary.

Nummular dermatitis can present with discreet, annular pruritic eczematous plaques, often with crusting.

The other common disorders in the differential diagnosis usually can be recognized and distinguished from AD based on characteristic signs, symptoms, and history.

Some findings and differences from typical atopic dermatitis disease course should prompt consideration of a broader differential diagnosis than atopic dermatitis, depending on a patient's age (summarized in Table 3). In an infant or young child, consideration of a differential diagnosis of rare disorders-metabolic, nutritional, genetic, immune, and proliferative conditions (Table 4) - becomes especially important when a growth curve abnormality/failure to thrive is noted, when multiple cutaneous and/or systemic infections occur, when the morphology or distribution of a rash is unusual, when a patient's response to typical AD treatment is poor, or when fixed-plaque hypopigmentation is treated and then recurs in the same site and in the same configuration, which suggests the possibility of cutaneous T-cell lymphoma.

In adults, late-onset $\mathrm{AD}$ signs and symptoms should prompt consideration of other diagnoses in addition to AD (Table 5).

TABLE 2 Differential Diagnosis of Atopic Dermatitis: Common Disorders

\begin{tabular}{l} 
Contact dermatitis (allergic and irritant) \\
Ichthyosis vulgaris \\
Keratosis pilaris \\
Nummular dermatitis \\
Psoriasis \\
Scabies \\
Seborrheic dermatitis \\
Tinea corporis \\
\hline \\
TABLE 3 Findings Which Should Prompt \\
Reconsideration of the Diagnosis of Atopic \\
Dermatitis in Infants and Young Children
\end{tabular}

Failure to thrive

Multiple infections, cutaneous and/or systemic

Unusual morphology or distribution of rash

Poor response to typical atopic dermatitis $(A D)$ treatments

Fixed-plaque hypopigmentation

Late-onset AD signs/symptoms

\section{Clinical Variations in AD in Skin of Color}

In patients with Fitzpatrick skin types IV, V, and VI, the cutaneous signs that are classic for AD in patients with lighter skin may not be evident (Figure). For example, erythema can be especially hard to detect on simple visual inspection (redness may be appreciated on skin blanching).

Lichenification and follicular accentuation are more common in skin of color, as are hypopigmentation and/or hyperpigmentation. In some cases, hypopigmentation can be profound, causing patients and families to be concerned that the change in skin coloration represents scarring and/or a side effect of a topical medication. They should be reassured that pigment changes associated with $\mathrm{AD}$ are common and are the result of inflammation in the skin.

In addition, certain features of $\mathrm{AD}$, such as xerosis, may have a different appearance in skin of color. The presence of some nonspecific findings that may be associated with AD can be helpful in making the diagnosis in patients with darker skin.

Pityriasis alba is commonly seen in individuals with dark skin, although it may occur in these patients in the absence of AD. Perifollicular accentuation and prurigo lesions also are common in patients with skin of color. Because filaggrin mutations are associated with hyperlinear palms and a tendency toward skin dryness, hyperlinearity suggests a diagnosis of AD.

TABLE 4 Rare Disorders to Be Considered in the Differential Diagnosis of Atopic Dermatitis in Infants and Children

\section{Metabolic/nutritional/genetic disorders \\ Acrodermatitis enteropathica}

Eosinophilic gastroenteritis

Gluten-sensitive enteropathy

Hurler syndrome

Netherton syndrome

Omenn syndrome

Other nutritional deficiencies (biotin, essential fatty acids)

Phenylketonuria

Prolidase deficiency

Zinc deficiency (prematurity; deficient breast milk zinc; cystic fibrosis)

Immune disorders

Agammaglobulinemia

Ataxia-telangiectasia

Hyperimmunoglobulin E syndrome

Neonatal lupus erythematosus

Severe combined immunodeficiency disorder

Wiskott-Aldrich syndrome

Proliferative disorders

Langerhans cell histiocytosis 
TABLE 5 Rare Disorders to Be Considered in the Differential Diagnosis of Atopic Dermatitis in Adolescents and Adults

Cutaneous T-cell lymphoma
(mycosis fungoides, Sézary syndrome)

Dermatomyositis

Drug eruptions

Graft-versus-host disease

HIV-associated dermatoses

Lupus erythematosus

Pemphigus foliaceus

HIV=human immunodeficiency virus.

\section{Assessment of AD}

Once the diagnosis of AD is established, an assessment of severity, persistence, and impact on the patient's and family's life is important as a guide to treatment decisions.

\section{Clinical Assessment}

Severity. About one-third of children with AD have severe disease, which can be predicted by three main factors: onset of signs and symptoms before 1 year of age, the presence of a filaggrin gene mutation, and concomitant immunoglobulin $\mathrm{E}$ (IgE) sensitization early in life. The degree of severity is judged on the basis of extent of involvement (the body surface area affected), qualities of the lesions, the persistence of the disease, and the impact of AD on a patient's and family's quality of life.

Persistence is defined by cycles of remission and relapse of signs and symptoms. Patients whose AD responds readily to standard treatment modalities and who experience prolonged periods of remission with occasional flares of signs and symptoms are considered to have less severe disease than those whose AD is difficult to bring under control and who relapse frequently.

Impact of disease. Clinicians should ask questions that probe quality-of-life challenges for the patient and the family, such as sleep disturbance, interference with school and/or work, effects on relationships, and disruption of family life.

\section{Assessment Tools and Laboratory Tests for AD}

Tests of AD severity that have been developed for use in clinical trials - the Eczema Area and Severity Index (EASI) and SCOring Atopic Dermatitis (SCORAD) - and patient assessment measures such as the Patient-Oriented Eczema Measure (POEM) and the Patient-Oriented SCOring Atopic Dermatitis (PO-SCORAD) take a great deal of time to use and may not be suitable for use in clinical practice.

Laboratory tests that are sometimes performed to assess AD - namely, IgG testing for food allergies and gluten sensitivity testing - are of no clinical value. In the past, some clinicians had advocated the use of allergen-specific IgE tests as part of an assessment for allergies that might be "causing the eczema," but more recent studies have demonstrated that these tests have poor predictive value, with a high probability of falsepositive results. Studies have shown IgE testing for food allergy to have poor predictive value, with skin-prick tests only marginally more useful. ${ }^{11,12}$ These tests may be useful when evaluating for specific food responses in suspected allergies.

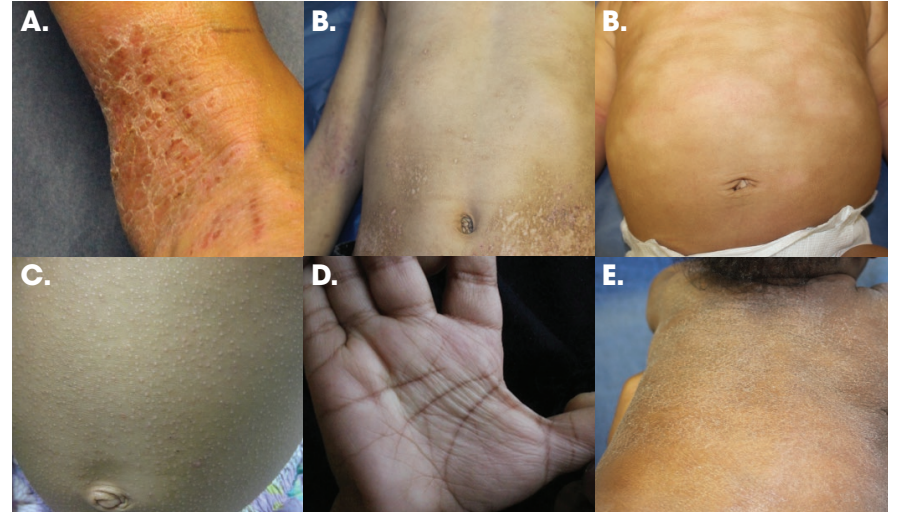

FIGURE Lichenification (A), Hypopigmentation (B), Perifollicular Accentuation (C), Palmar Hyperlinearity (D), Xerosis (E)

Source: Photos courtesy of Lawrence F. Eichenfield, MD.

\section{Follow-Up Evaluation: Practical Questions}

At follow-up visits, useful information can be obtained about recent routines for skin care and medication use during the previous week, rather than just asking about use since the last visit. In assessing topical medication use, it is often helpful to ask how long a tube of the patient's medication lasts.

Other questions should elicit information relating to the period since the last visit, including the last time the patient's skin was totally clear, whether any systemic medications (such as oral prednisone) have been prescribed by another clinician, and whether the patient has been hospitalized for any reason or has been seen by a clinician in another specialty, such as an allergist.

Finally, each clinical interaction should include an assessment of how the patient and/or the family think about AD. The simple question "What are you afraid of concerning this skin condition?" can elicit a focused answer with useful information.

\section{Conclusion}

Atopic dermatitis is a common condition that is usually easy to diagnose clinically, although considerations of a broader differential diagnosis are appropriate when the history, clinical course, or response to therapy are atypical. Evaluations of AD should include assessment of disease severity, persistence, response to therapies, and disease impact on the individual and family.

\section{References}

1. Eyerich K, Novak N. Immunology of atopic eczema: Overcoming the Th1/Th2 paradigm. Allergy. 2013;68:974-982.

2. Leung DY, Bieber T. Atopic dermatitis. Lancet. 2003:361:151-160.

3. Blumberg SJ, Olson L, Frankel M, Osborn L, Srinath KP, Giambo P. Design and operation of the National Survey of Children's Health 2003 (NSCH). Vital Health Stat 1. 2005;(43):1-124. [National Survey of Children's Health (NSCH), 2003 www. childhealthdata.org].

4. Simpson EL, Hanifin JM. Atopic dermatitis. Med Clin North Am. 2006;90:149-167.

5. Ozkaya E. Adult-onset atopic dermatitis. J Am Acad Dermatol. 2005;52:579-582.

6. Shaw TE, Currie GP, Koudelka CW, Simpson EL. Eczema prevalence in the United States: Data from the 2003 National Survey of Children's Health. J Invest Dermatol. 2011;131:67-73.

7. Kathuria P, Silverberg JI. Association of pollution and climate with atopic eczema in US children. Pediatr Allergy Immunol. 2016;27:478-485.

8. Siegfried EC, Hebert AA. Diagnosis of atopic dermatitis: Mimics, overlaps, and complications. J Clin Med. 2015;4:884-917.

9. Weidinger S, Novak N. Atopic dermatitis. Lancet. 2016;387:1109-1122.

10. Eichenfield LF, Tom WL, Chamlin SL, et al. Guidelines of care for the management of atopic dermatitis: Section 1. Diagnosis and assessment of atopic dermatitis J Am Acad Dermatol. 2014;70:338-351.

11. Spergel JM, Boguniewicz M, Schneider L, Hanifin JM, Paller AS, Eichenfield LF. Food allergy in infants with atopic dermatitis: Limitations of food-specific IgE measurements. Pediatrics. 2015;136.

12. Du Toit G, Roberts G, Sayre PH, et al. Learning Early About Peanut Allergy (LEAP) Study Team. Identifying infants at high risk of peanut allergy: The Learning Early About Peanut Allergy (LEAP) screening study. J Allergy Clin Immunol. 2013;131:135-143. 